\title{
Inkjet-printed Meta-material Inspired Passive Antenna Sensor for UHF RFID Systems
}

\author{
${ }^{\dagger}$ Apostolos Georgiadis and Ana Collado \\ Centre Tecnologic de Telecomunicacions de Catalunya \\ CTTC \\ Barcelona, Spain \\ †apostolos.georgiadis@cttc.es
}

\author{
\$Sangkil Kim and Manos M. Tentzeris \\ School of Electrical and Computer Engineering \\ Georgia Institute of Technology \\ Atlanta, GA, USA \\ †kangkil3@gatech.edu
}

\begin{abstract}
A fully passive low-cost wireless UHF RFID antenna based sensor for Internet of Things applications is presented. The proposed sensor consists of a closely spaced two-element dipole antenna with a printed sensor electrically connected to one of the antennas. A metamaterial-inspired resonator is used to improve isolation between the two antennas and optimize the size of the antenna structure. The radiating and sensing structure is inkjetprinted on photo-paper using a silver nanoparticle ink, and commercial RFID chips are connected to each of the two antenna terminals. The tag dimensions are $84 \mathrm{~mm} \times 89 \mathrm{~mm}$ and the tag is compatible with an EPC Class 1 Gen 2 (UHF) standard reader operating at $915 \mathrm{MHz}$. The design methodoly of the sensor as well as theoretical analysis and experimental results are presented.
\end{abstract}

Keywords-Dual-tag sensing, haptic sensor, inkjet-printing technology, Internet of Things (IoT), remote sensing, RFID-enabled sensor, wireless sensors, antenna based sensor.

\section{INTRODUCTION}

RFID-enabled sensors are receiving significant scientific interest due to the fact that they combine the advantages of RFID technology such as low profile and simple circuit architecture, low power dissipation, and wireless powering capability allowing battery-less operation, with sensing features such as temperature, gas, strain and humidity. Consequently they find numerous applications related to smart sensing and the Internet of Things [1].

Antenna based sensing [2] allows for a simple and passive way of integrating sensing capabilities to existing RFID systems. A passive sensor transducer modifies an electrical parameter such as resistance, capacitance or inductance based on a sensing property, which in turn changes the load of the tag antenna and RFID chip system and thus alters the back-scattered signal from the RFID tag in a unique way which can be interpreted by the reader as a change in a desired sensing parameter. In [3] the concept of introducing a dual antenna sensor has been proposed, where the response of a second tag placed in close proximity to the sensor tag is used as a reference to help detect the sensing parameter. The challenge in such topologies is to maintain a low profile by minimizing the size of the sensor/antenna system, and at the same time to minimize the coupling between the reference and sensing tags in order to maintain the detection capability.

Furthermore, the use of additive manufacturing methods such as inkjet printing presents a competitive alternative to

The work of S. Kim and M. M. Tentzeris was supported by National Science Foundation (NSF) and Defense Threat Reduction Agency (DTRA). The work of A. Georgiadis and A. Collado was supported by the Spanish Ministry of Economy and Competitiveness and FEDER funds through project TEC2012-39143 and by the Generalitat de Catalunya under grant 2014 SGR 1551. traditional milling or chemical etching, and has been successfully used to fabricate antennas and passive structures at UHF frequencies and on a variety of substrate materials such as paper [1].

This paper presents the design of a dual tag passive sensing system, inkjet printed on paper. The dimensions of the tag dipole antennas and their coupling have been optimized by introducing a resonance with the help of a printed inductive and capacitive structure within the dipole arms, similarly to the structure proposed in [4] for monopole antennas. The length of the proposed inkjet-printed antenna on paper substrate is $89.2 \mathrm{~mm}$ $(0.27 \lambda 0)$ which is $28.4 \%$ shorter than the length of the inkjetprinted dipole antenna on paper substrate without any miniaturization $(125 \mathrm{~mm},[5])$ at the operation frequency of 915 $\mathrm{MHz}$. Preliminary results of a prototype have been presented in [6]. In this paper, we emphasize on the read range analysis and measured performance of the sensor.

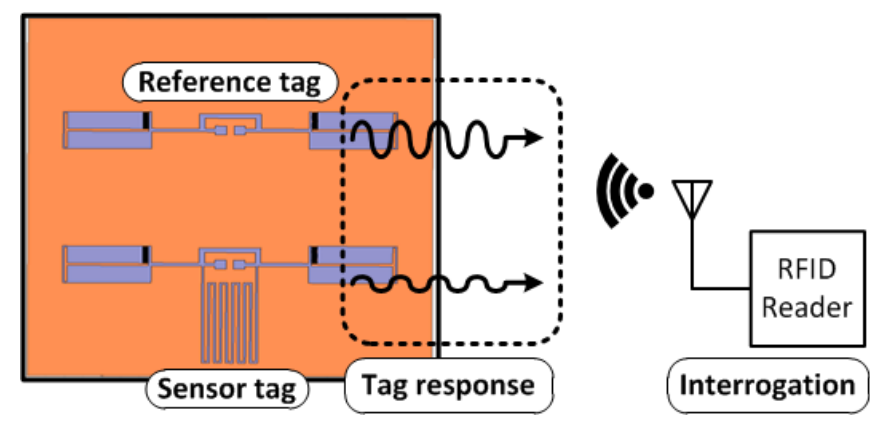

Fig. 1. RFID-enabled dual-tag sensor topology

\section{Dual RFID Tag SEnsing \& Antenna Design}

\section{A. Dual-tag Sensing Topology}

The concept of the dual tag sensor is pictured in Fig. 1. A commercial reader interrogates simultaneously the reference and sensing tags. The reference tag is a dipole tag, while the sensing tag additionally includes a meandered loop which acts as proximity or touch sensor. The size of the dipole antennas as well as the coupling between them has been reduced by introducing an LC resonance by printing an interdigitated capacitor and slot shown in Fig. 2. 


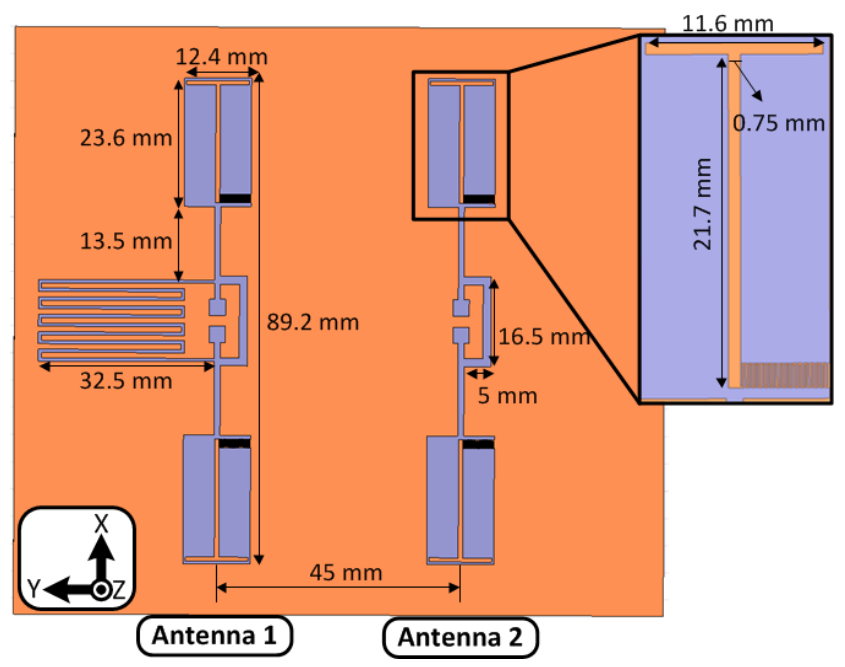

Fig. 4. Geometry of the proposed dual-tag RFID enabled sensor.

The effect of the additional resonance on the coupling between the two antennas is further shown in Fig. 3 and Fig. 4. In Fig. 3 the electric field distribution on the two tag antennas is shown with and without the LC resonator when one of the two antennas is excited (Antenna 1). A reduction in the field magnitude at the second antenna (Antenna 2) is observed. Correspondingly, a reduction by approximately $3 \mathrm{~dB}$ in the $\mathrm{S}_{21}$ magnitude has been observed (Fig. 4).

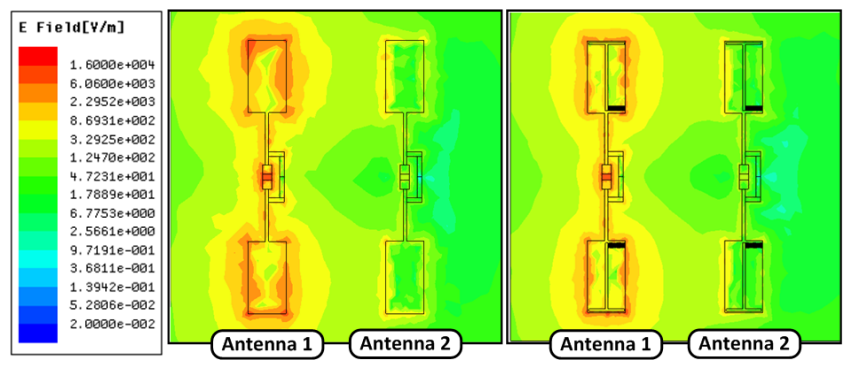

Fig. 3. E-field distribution of the sensor tag (a) without the printed LC resonators and (b) with the printed $\mathrm{LC}$ resonators.

\section{READ RANGE ANALYSIS}

The effect of the sensing event in the read range of the two antenna system has been studied by considering free-space propagation and a two-port loaded scatterer system. It can be derived as a specific case of the general analysis of RFID grids of $\mathrm{N}$ tags developed in [7]. The read range $\mathrm{R}_{n}$ of tag $\mathrm{n}=1,2$ can be expressed in a similar manner as for the single (1 port) tag antenna [8] as

$$
\frac{\mathrm{R}_{\mathrm{n}}}{\mathrm{R}_{0}}=\sqrt{\mathrm{G}_{\mathrm{n}} \tau_{\mathrm{n}}}
$$

where the the gain $G_{n}$ and power transmission coefficient $\tau_{n}$ include the effect of coupling between the two antennas. The gain $G_{n}$ is computed with the help of a commercial simulator by exciting the port antenna $\mathrm{n}$ and leaving the other port open. The reference range factor $R_{o}$ depends on the wavelength, the tag minimum activation power $P_{\text {th }}$ and the reader gain $G_{t}$ and transmitted power $P_{t}[8]$ :

$$
\mathrm{R}_{0}=\frac{\lambda_{0}}{4 \pi} \sqrt{\frac{\mathrm{P}_{\mathrm{t}} \mathrm{G}_{\mathrm{t}}}{\mathrm{P}_{\mathrm{th}}}}
$$

The gain $G_{n}$ and power transmission coefficient $\tau_{n}$ can be written as

$$
\begin{gathered}
G_{n}=\frac{4 \pi}{\eta R_{A n}}\left|F_{n}\right|^{2}=\frac{\left|g_{n}\right|^{2}}{R_{A n}} \\
\tau_{n}=4 R_{c} R_{A n} \frac{\left|[Y]_{n} g\right|^{2}}{\left|g_{n}\right|^{2}}
\end{gathered}
$$

where $\mathbf{Y}=\left(\mathbf{Z}_{\mathbf{C}}+\mathbf{Z}_{\mathbf{A}}\right)^{-1}$ with $\mathbf{Z}_{\mathbf{A}}=\mathbf{R}_{\mathbf{A}}+j \mathbf{X}_{\mathbf{A}}$ the impedance matrix of the 2-port antenna system and $Z_{c}$ the tag chip impedance $\left(\mathbf{Z}_{\mathrm{C}}=\mathrm{Z}_{\mathrm{c}} \mathbf{I}_{\mathbf{2}}\right.$ with $\mathbf{I}_{\mathbf{2}}$ the identity matrix of dimension n). $[\mathbf{Y}]_{n}$ denotes the $\mathrm{n}$-th row (vector) of matrix $\mathbf{Y}$. Finally, $\mathbf{g}=$ $\left[g_{n}\right]$ and $F_{n}$ is the radiated field $F_{n}$ of the antenna system when a unit current excitation is applied at port $n$ with the other port open.

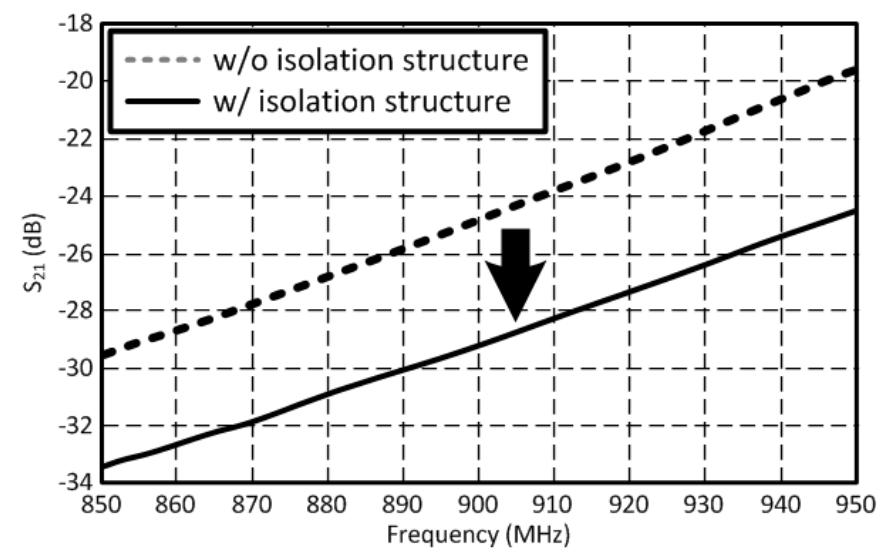

Fig. 4. Magnitude of crosstalk between the antenna 1 and the antenna 2 with/without the printed LC resonators.

After a long but straightforward calculation the result of Fig. 5 is obtained where the concept of the proposed sensor is demonstrated. The range of a single (1-tag) and that of the proposed 2-tag antenna sensor are estimated in the case of no event and in the case where an object is touching the sensor. The simulated object is a human finger modeled as a rectangular box which has the dielectric permittivity of 21.14 and the conductivity of $0.36 \mathrm{~S} / \mathrm{m}$. The values of the dielectric constant and the conductivity were chosen to the mean values of those of a human skin, a flesh, and a bone [9]. One can see that in the case of the 1-tag system, the presence of the object leads to a strong effect in the antenna gain and read range. In the case of the two tag system however, only the sensing tag is strongly affected by the object. 


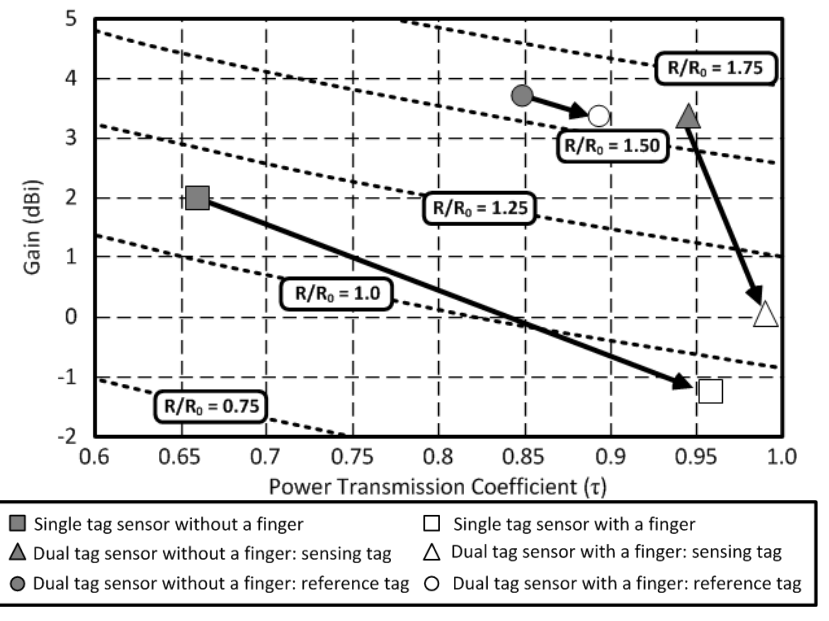

Fig. 5. Read range analysis of the RFID-enabled sensor.

\section{MEASUREMENT}

Read range measurements have been performed using a Voyantic Tagformance system [10]. The result of Fig. 6 shows the delivered power at each tag obtained from the measurement setup for $30 \mathrm{dBm}$ transmitted power and $0.6 \mathrm{~m}$ interrogation distance, where one can also verify that in the case of no event (no object in proximity) the performance of the two tags comprising the sensor is similar, while the presence of the object has a stronger effect in the sensing tag than the reference tag.

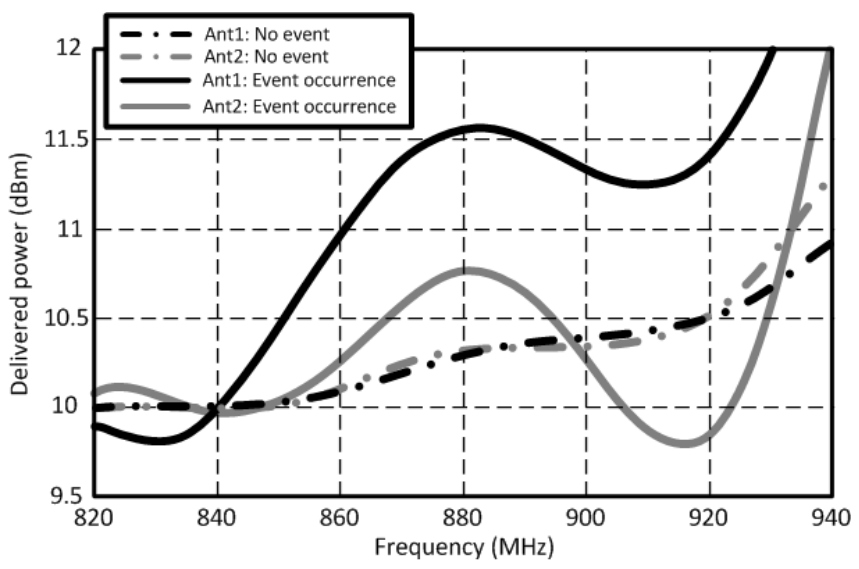

Fig. 6. Delivered power to each antenna.

\section{SUMMARY}

In this paper, an inkjet-printed dual-tag RFID-enabled haptic sensor with meta-material inspired LC resonators, which are embedded in the tag antennas, are implemented on paper substrates. The dual-tag sensing topology has numerous advantages such as less-environment dependent operation due to differential detection method. The next step of this work is to develop a reader to implement a robust detection scheme.

\section{ACKNOWLEDGMENT}

The work of S. Kim and M. M. Tentzeris was supported by National Science Foundation (NSF) and New Energy and Industrial Technology Development Organization (NEDO). The work of A. Georgiadis and A. Collado was supported by the Spanish Ministry of Economy and Competitiveness project TEC2012-39143 and by EU Marie Curie project FP7-PEOPLE2009-IAPP 251557.

\section{REFERENCES}

[1] A. Rida, L. Yang and M. Tentzeris, RFID-Enabled Sensor Design and Applications, Artech House, 2010.

[2] R. Bhattacharyya, C. Floerkemeier, and S. Sarma, "Towards tag antenna based sensing - An RFID displacement sensor," 2009 IEEE International Conference on RFID, pp.95-102, 27-28 April, 2009.

[3] S. Johan, Z. Xuezhi, T. Unander, A. Koptyug, and H. E. Nilsson, "Remote Moisture Sensing utilizing Ordinary RFID Tags," IEEE Sensors, pp.308311, 28-31 Oct. 2007.

[4] J. Zhu, and G. V. Eleftheriades, "A simple approach for reducing mutual coupling in two closely spaced metamaterial-inspired monopole antennas," IEEE Antennas Wireless Propag. Lett., vol.9, pp.379-382, Apr. 2010.

[5] H. Lee, S. Kim, D. De Donno, and M. M. Tentzeris, "A novel "Universal" inkjet-printed EBG-backed flexible RFID for rugged on-body and metal mounted applications," IEEE MTT-S International Microwave Symposium Digest (MTT), Montréal, Canada, Jun. 2012, pp.1-3.

[6] S. Kim, Y. Kawahara, A. Georgiadis, A. Collado, and M. M. Tentzeris, "Low-cost inkjet-Printed fully passive RFID tags using metamaterialinspired antennas for capacitive sensing applications," 2013 IEEE MTTS International Microwave Symposium (IMS), Seattle 2-7 June 2013.

[7] G. Marrocco, "RFID grids: Part I-Electromagnetic theory," IEEE Trans. Antennas Propag., vol.59, no.3, pp. 1019-1026, Mar. 2011.

[8] K. V. S. Rao, P. V. Nikitin, and S. F. Lam, "Antenna design for UHF RFID tags: a review and a practical application," IEEE Trans. Antennas Propag., vol.53, no.12, pp.3870-3876, Dec. 2005.

[9] C. Gabriel, "Compilation of the dielectric properties of body tissues at RF and microwave frequencies", Brooks ir Force Technical Report AL/OETR-1996-0037, 1996.

[10] [Online] Available: http://www.voyantic.com/tagformance. 